\title{
Avaliação da densidade de potência e do espectro de luz de fotoativadores usados em consultórios odontológicos
}

\section{Evaluation of the power density and the light spectrum of light from light-curing units used in dental clinics}

\author{
Ricardo Danil GUIRALDO \\ Mestre em Materiais Dentários pela Faculdade de Odontologia de Piracicaba - Universidade Estadual de Campinas - \\ UNICAMP - Piracicaba - SP - Brasil
}

\section{Simonides CONSANI \\ Mario Alexandre Coelho SINHORETI \\ Lourenço CORRER-SOBRINHO}

Professor Titular da área Materiais Dentários da Faculdade de Odontologia de Piracicaba - Universidade Estadual de Campinas - UNICAMP - Piracicaba - SP - Brasil

\section{Rafael Leonardo Xediek CONSANI}

Professor Adjunto do Departamento de Prótese e Periodontia da Faculdade de Odontologia de Piracicaba - Universidade Estadual de Campinas - UNICAMP - Piracicaba - SP - Brasil

\section{Ana Paula Piovezan FUGOLIN \\ Aluna de Graduação, Faculdade de Odontologia de Piracicaba - Universidade Estadual de Campinas - UNICAMP - Piracicaba - SP - Brasil}

\begin{abstract}
Resumo
O estudo avaliou a densidade de potência e o espectro de diferentes fotoativadores usados em consultórios odontológicos de Piracicaba, SP. A potência (mW) dos fotoativadores foi mensurada com potenciômetro (Ophir 10A-V2-SH; Ophir Optronics). O diâmetro da ponta do aparelho fotoativador foi calculado e a área de emissão de luz mensurada $\left(\pi r^{2}\right)$. Posteriormente verificou-se a densidade de potência (irradiância) por meio de cálculo matemático (potência/ área). O espectro de luz emitido pela fotoativador foi obtido com espectrômetro (USB 2000; Ocean), para a caracterização dos espectros e mensuração da irradiância conforme a ISO/TS:10650 por meio de cálculos integrais das áreas nas regiões: 400 a $515 \mathrm{~nm}, 190$ a 400nm e acima de $515 \mathrm{~nm}$. Os consultórios foram classificados de acordo com o preço praticado para uma face de restauração de compósito: valor mínimo de $\mathrm{R} \$ 50,00$ (consultório nível C); de $\mathrm{R} \$ 50,00$ até $\mathrm{R} \$ 70,00$ (nível B); acima de R \$70,00 (nível A); consultório de serviço público; e de planos odontológicos. Na região de 400-515nm, o nível A apresentou $30 \%$ de fotoativadores abaixo de $300 \mathrm{~mW} / \mathrm{cm}^{2}$; o nível B e clínicas de convênios apresentaram $50 \%$. O nível C registrou 40\%, e serviço público mostrou 20\%. Na região 190-400nm não foi verificado fotoativadores fora da norma, em nenhum nível. Para a região acima de 515nm, o nível A mostrou 20\% e os níveis B e C registraram 10\% dos fotoativadores acima de $50 \mathrm{~mW} / \mathrm{cm}^{2}$. Em todos os níveis, a densidade de potência dos fotoativadores não estava de acordo com o recomendado pela ISO/TS: 10650.
\end{abstract}

\section{UNITERMOS}

Fotoativadores; densidade de potência; espectro de luz; irradiância; consultórios odontológicos.

\section{INTRODUÇÃo}

Em meados dos anos 60 foram introduzidos no mercado odontológico compósitos com indicação para dentes anteriores, em substituição aos restauradores estéticos cimento de silicato e resina acrílica². No entanto, os compósitos fotoativados foram introduzidos no mercado somente na década de 70 . Os primeiros 
produtos eram fotoativados por luz ultravioleta, que oferecia riscos à visão, tanto do operador quanto do paciente e proporcionava ao compósito propriedades físicas e mecânicas insatisfatórias ${ }^{20,24}$. Como conseqüência, versões posteriores passaram a ser fotoativadas por luz visível ${ }^{23}$. Para o uso dessas fontes foi necessário o desenvolvimento de compósitos restauradores apropriados, os quais passaram a conter fotoiniciadores sensíveis à luz visível.

Os aparelhos mais tradicionais com emissão de luz visível são constituídos por lâmpada de quartzo-tungstênio-halogênio (também conhecida como lâmpada halógena) ${ }^{9}$. Essas lâmpadas contêm um filamento de tungstênio conectado a eletrodos, o qual permite o fluxo da eletricidade, gerando luz e calor ${ }^{22}$. As lâmpadas de quartzo-tungstênio-halogênio emitem luz branca que ao passar por filtros específicos seleciona determinadas regiões do espectro eletromagnético. Desta forma, apenas a região azul do espectro é selecionada para a fotoativação do compósito odontológico ${ }^{3}$, região de absorção da canforoquinona que é considerado o fotoiniciador mais utilizado na composição das resinas compostas, com espectro de absorção no intervalo entre 400 e $500 \mathrm{~nm}$. O comprimento de onda mais eficiente para a polimerização seria no intervalo de 468 - 470nm ${ }^{18}$.

O diodo emissor de luz (LED) foi utilizado para fotopolimerizações com o objetivo de minimizar o calor gerado durante a fotoativação pela luz halógena $^{27}$. O LED emitindo comprimento de onda de 455 a 486nm se relaciona com a taxa de absorção do espectro da canforoquinona ${ }^{19}$. Assim, o espectro de emissão de uma fonte de luz possui forte influência no desempenho da fotoativação ${ }^{16}$.

Tempos de exposição prolongados para fotoativação são desconfortáveis para os pacientes, especialmente pediátricos e inconvenientes para os profissionais por tomar maior tempo clínico para a realização dos procedimentos de restauração e cimentação. Para reduzir o tempo de fotoativação, os aparelhos de arco de plasma de xenônio foram introduzidos no mercado odontológico, propondo a utilização de fonte de luz que gerasse alta intensidade. A luz é emitida por um plasma incandescente, o qual é composto por uma mistura gasosa de moléculas ionizadas e elétrons ${ }^{12,20}$.

Além da correlação entre o espectro emitido pela fonte de luz e o espectro de absorção do fotoiniciador, o processo de fotoativação é dependente de outros fatores como, por exemplo, a densidade de potência $\left(\mathrm{mW} / \mathrm{cm}^{2}\right)$ emitida pela fonte de luz e o tempo de exposição. Caso o compósito não receba quantidade suficiente de densidade de energia, o grau de conversão monomérico será baixo ${ }^{17}$, resultando em possível aumento da citotoxicidade ${ }^{4}$, desgaste e quebra de margens ${ }^{8}$, assim como redução da dureza e do módulo de elasticidade ${ }^{11}$.

O espectro emitido pela fonte de luz, a intensidade da luz emitida e o modo de fotoativação são fatores importantes quando associados à efetividade de fotoativação das fontes de luz ${ }^{21,22}$. Entretanto, a maioria dos estudos ${ }^{5,26}$ que comparam o desempenho de diversas fontes de luz não verificam o espectro emitido pelas fontes de luz. Além disto, utilizam apenas radiômetros para verificar a intensidade $\left(\mathrm{mW} / \mathrm{cm}^{2}\right)$ das mesmas. No entanto, esses métodos mostram apenas a intensidade total emitida pela fonte de luz e trazem informações limitadas sobre diferenças existentes no desempenho de diferentes fontes de luz, dependentes dos radiômetros utilizados. No entanto, parece haver pouca consistência nos resultados encontrados em radiômetros comumente utilizados nos consultórios odontológicos ${ }^{6,10,25}$. Além disso, caso a emissão do espectro da fonte de luz seja registrada por meio de espectrômetro, pode-se mensurar a densidade de potência da luz em função do comprimento de onda por meio de cálculo integral da área ${ }^{21}$.

Diante destas considerações, o estudo teve como objetivo avaliar a densidade de potência e o espectro de luz de diferentes foativadores usados em consultórios odontológicos, considerando o preço praticado no consultório avaliado. A hipótese deste estudo foi que consultórios com clientes de classe social econômica maior usariam fotoativadores mais eficientes.

\section{Materiais e Método}

Este estudo foi realizado na cidade de Piracicaba, SP, onde foram avaliados 50 consultórios odontológicos para mensuração dos fotoativadores, com a intenção de verificar se essas unidades fotoativadoras emitiam a densidade de potência mínima necessária para promover polimerização eficiente dos compósitos. Inicialmente foram verificadas as condições das fontes de luz utilizadas pelos Cirurgiões Dentistas. Em seguida, foi avaliada a densidade de potência $\left(\mathrm{mW} / \mathrm{cm}^{2}\right)$ e calculado os espectros emitidos pelas fontes de luz.

O diâmetro da ponta de saída da luz do aparelho foi mensurado com paquímetro digital (Digital caliper, model CD-15C, Mitutoyo, Japan) para determinar a área de emissão da luz $\left(\pi \mathrm{r}^{2}\right)$. A potência $(\mathrm{mW})$ das fontes de luz foi mensurada com potenciômetro (Ophir 
10A-V2-SH; Ophir Optronics, Har - Hotzvim, Jerusalém, Israel) acoplado a um microprocessador (NOVA; Ophir Optronics).

Com estes dados foi possível determinar a densidade de potência por meio da fórmula: Densidade de potência $\left(\mathrm{mW} / \mathrm{cm}^{2}\right)=$ Potência $(\mathrm{mW}) /$ Área $\left(\mathrm{cm}^{2}\right)$.

As distribuições dos espectros emitidos pelas fontes de luz foram obtidas com auxílio de espectrômetro (USB 2000; Ocean Optics, Dunedin, FL, EUA), com corretor cossenoidal conectado ao computador.

Os valores de densidade de potência $\left(\mathrm{mW} / \mathrm{cm}^{2}\right)$ e dos espectros emitidos foram transferidos para o programa Origin 6.1 (OriginLab Corp., Northampton, MA, EUA) para obter, por meio de cálculos integrais da área, os valores de densidade de potência nas seguintes regiões do espectro:

1. Entre 400-515nm: não deve ser menor do que $300 \mathrm{~mW} / \mathrm{cm}^{2}$ nem maior do que $1.000 \mathrm{~mW} / \mathrm{cm}^{2}$.

2. Entre 190-400nm: não deve exceder $100 \mathrm{~mW} /$ $\mathrm{cm}^{2}$.

3. Acima de $515 \mathrm{~nm}$ : não deve exceder $50 \mathrm{~mW} / \mathrm{cm}^{2}$.

O objetivo da aferição foi verificar se as fontes se enquadravam nas normas preconizadas pela ISO/TS: $10650^{13}$ e para auxiliar no entendimento dos resultados observados.

Os consultórios foram agrupados em diferentes níveis de classe social econômico, de acordo com o preço praticado nos procedimentos. O critério básico para classificação dos consultórios foi o preço por face de uma restauração de compósito, já que não existe uma classificação disponível bem definida. Até o valor mínimo estipulado em $\mathrm{R} \$ 50,00$, o consultório foi considerado nível $\mathrm{C}(\mathrm{n}=10)$. De $\mathrm{R} \$ 50,00$ até $\mathrm{R} \$ 70,00$, nível $B(n=10)$. Acima de $R \$ 70,00$, nível A $(n=10)$.
Também foram avaliados consultórios de serviço público $(n=10)$ e consultórios de diferentes planos odontológicos $(n=10)$. Os dados foram submetidos à análise descritiva baseada em porcentagem.

\section{Resultados}

A Tabela 1 mostra que na região entre $400-515 \mathrm{~nm}$, $30 \%$ dos consultórios considerados nível A, 50\% nível B, $40 \%$ nível C, $50 \%$ dos consultórios de convênios e $20 \%$ dos consultórios públicos tiveram equipamentos com intensidade de luz inferior a $300 \mathrm{~mW} / \mathrm{cm}^{2}$; e não houve em qualquer nível nenhum equipamento com intensidade superior a $1.000 \mathrm{~mW} / \mathrm{cm}^{2}$. Na região entre 190-400nm não houve em qualquer nível nenhum equipamento com intensidade superior a $100 \mathrm{~mW} / \mathrm{cm}^{2}$. Na região acima de 500nm, $20 \%$ do nível A, $10 \%$ dos níveis B e C excederam $100 \mathrm{~mW} / \mathrm{cm}^{2}$.

\section{Dıscussão}

A fotoativação realizada com baixos valores de intensidade de luz resulta em menor número de radicais livres gerados ${ }^{1}$. Este fato pode estar associado com menores densidades de ligações cruzadas, as quais seriam evidenciadas clinicamente pelo aumento da susceptibilidade à degradação da restauração ${ }^{7}$. A hipótese deste estudo que consultórios com clientes de classe social econômica maior usariam fotoativadores mais eficientes não foi comprovada.

Seria de se esperar que consultórios com prática de preço pelos procedimentos com maior valor econômico teriam equipamentos com maior eficiência fotoativadora. Entretanto, a qualidade da luz necessária para produzir polimerização eficiente dos compósitos odontológicos não estaria relacionada com o preço de

Tabela 1 - Porcentagem de equipamentos fora dos padrões ISO/TS:10650.

\begin{tabular}{|c|c|c|c|c|}
\hline Níveis & $\begin{array}{c}400-515 \mathrm{~nm} \\
\text { (menor que } \\
300 \mathrm{~mW} / \mathrm{cm}^{2} \text { ) }\end{array}$ & $\begin{array}{c}400-515 \mathrm{~nm} \\
(\text { maior que } \\
\left.1000 \mathrm{~mW} / \mathrm{cm}^{2}\right)\end{array}$ & $\begin{array}{c}190-400 \mathrm{~nm} \\
(\text { excesso de } \\
\left.100 \mathrm{~mW} / \mathrm{cm}^{2}\right)\end{array}$ & $\begin{array}{c}\text { Acima de 500nm } \\
\text { (excesso de } \\
\left.100 \mathrm{~mW} / \mathrm{cm}^{2}\right)\end{array}$ \\
\hline Nível A & $30 \%$ & $0 \%$ & $0 \%$ & $20 \%$ \\
\hline Nível B & $50 \%$ & $0 \%$ & $0 \%$ & $10 \%$ \\
\hline Nível C & $40 \%$ & $0 \%$ & $0 \%$ & $10 \%$ \\
\hline Consultórios de Convênios & $50 \%$ & $0 \%$ & $0 \%$ & $0 \%$ \\
\hline Consultórios públicos & $20 \%$ & $0 \%$ & $0 \%$ & $0 \%$ \\
\hline
\end{tabular}


mercado do fotoativador. O preço do fotoativador estaria na dependência do custo tecnológico da unidade fotoativadora, que nem sempre está relacionada com a eficiência em uso. No presente estudo foi considerada a qualidade da luz que os fotoativadores emitiam e não houve relação positiva entre maior preço cobrado por procedimento e melhor qualidade da luz emitida pelos fotoativadores. Os resultados deste estudo mostram um achado altamente relevante, indicando que os clínicos não precisam adquirir unidades fotoativadoras de maior preço para produzir eficiente polimerização das resinas.

Em todos os níveis foram encontrados fotoativadores fora da norma ISO/TS: $10650^{13}$. Rueggberg ${ }^{22}$ (1999) relata que somente $9 \%$ da intensidade de luz que incide sobre a superfície do compósito atinge a base em um incremento de $2 \mathrm{~mm}$ de espessura. Este fato poderia acarretar propriedades mecânicas indesejadas ao compósito na porção da base da restauração. Numa restauração tipo classe II, a área de adesão substrato dental-resina composta seria na proximal (mais profundo em relação à fonte de luz), onde ocorreria a falha que pode resultar em cáries recorrentes e até mesmo uma lesão pulpar irreversível.

A ativação dos compósitos por luz visível também pode contribuir para aumentar a temperatura no interior da câmara pulpar, causando possíveis danos à polpa $^{14,15}$. O aumento da intensidade da luz pode elevar a temperatura durante a polimerização, devido à maior energia de radiação fornecida pelo aparelho fotoativador ${ }^{10}$. Neste estudo, nenhum dos níveis classificatórios apresentou aparelhos com intensidade superior a 1000 $\mathrm{mW} / \mathrm{cm}^{2}$ dentro da região entre $400-515 \mathrm{~nm}$.

Dentre as unidades fotoativadoras disponíveis no mercado, as mais tradicionais são as que utilizam lâmpadas halógenas como fonte de luz ${ }^{9}$. Contudo, a principal irradiação produzida por essas lâmpadas é o espectro infravermelho, o qual é absorvido pelo compósito resultando grande vibração molecular e geração de calor ${ }^{26}$. Assim, fontes de luz que utilizam lâmpadas halógenas necessitam de filtros termo-absorventes que reduzem a passagem da energia infravermelha para o dente ${ }^{22}$. No entanto, a eficiência destes filtros varia conforme cada fabricante e, assim, a energia não absorvida pode não ser similar, resultando em diferentes níveis de calor.

$\mathrm{Na}$ região entre 190-400nm a intensidade de luz não deve exceder $100 \mathrm{~mW} / \mathrm{cm}^{2}$, região na qual se relaciona com a região ultravioleta do espectro de luz. Nenhum aparelho fotoativador mostrou-se não atender a norma ISO/TS: $10650^{13}$. Nesta região a luz oferece riscos à visão tanto de operador quanto ao paciente. Por essa razão, este achado se torna de grande valia devido ao fato que tanto profissionais quanto pacientes não estão sujeitos à sérios riscos de danos visuais.

No presente estudo, na região acima de $515 \mathrm{~nm}$ foi observado que $20 \%$ no nível A e $10 \%$ nos níveis $\mathrm{B}$ e $\mathrm{C}$ das unidades fotoativadoras excediam $50 \mathrm{~mW} /$ $\mathrm{cm}^{2}$ apenas naqueles fotoativadores que utilizavam a luz halógena. Desta maneira, esta região se relaciona com a região infravermelha do espectro da luz, ou seja, região onde existe formação de calor. Este fato poderia aumentar a temperatura do dente, causando possíveis danos à polpa ${ }^{14,15}$. Além da ineficiência dos filtros, este fato também pode estar relacionado com danos dos filtros termo-absorventes devido à quedas acidentais ou/e mau conservação do aparelho fotoativador.

Este estudo sugere que a classe sócio-econômica não interfere na qualidade da unidade fotoativadora e que em todos os níveis de consultório existem fotoativadores que não se enquadram na norma ISO/ TS: $10650^{13}$.

\section{Conclusão}

Em todos os níveis de consultórios, a densidade de potência dos fotoativadores não se encontra de acordo com o recomendado pela ISO/TS: 10650.

\section{Abstrac}

The study evaluated the power density and light spectrum of different light curing unit (LCU) used in dental clinics of Piracicaba, SP. The power ( $\mathrm{mW}$ ) of the LCU was measured with power meter Ophir Optronics (Ophir 10A-V2-HS; Ophir Optronics). The diameter of the tips was measured with a digital caliper to determine the tip áreas $\left(\pi r^{2}\right)$. A mathematical calculation (power / area) was performed to determine the power density (irradiance). A spectrometer (USB 2000, Ocean Optics) was used to record the LUC light spectrum and then characterize and measure its irradiance according to ISO/ TS: 10650 by calculating the integral areas in the regions from 400 to $515 \mathrm{~nm}, 190$ to $400 \mathrm{~nm}$, and above $515 \mathrm{~nm}$. Dental clinics were classified according to the price charged for a restoration (one face): minimum value of R $\$ 50.00$ (level C); from R $\$ 50.00$ to R 70.00 (level B); above R 70.00 (level A); public service clinics; and dental insure clinics. In the 
region from 400-515nm, LCU was observed to be below 300mW/cm² for levels A (30\%), B (50\%), C (40\%), public service clinics (20\%), and dental insure clinics (50\%). In the region from 190-400nm, concernig all levels, LCU was found to be in accord with the norm. In the region over $515 \mathrm{~nm}$, LCU was observed to be over $50 \mathrm{~mW} / \mathrm{cm}^{2}$ for levels A (20\%), B (10\%), and C (10\%). At all levels, power density of LCU was not in accordance with that recommended by ISO/TS: 10650.

\section{UNITERMS}

Light curing unit; power density; light spectrum; irradiance; dental clinics.

\section{REFERÊNCIAS}

1. Asmussen E, Peutzfeldt A. Influence of pulse-delay curing on softening of polymer structures. J Dent Res. 2001;80(6):1570-3.

2. Bowen RL. Properties of silica-reinforced polymer for dental restorations. J Am Dent Assoc. 1963;66(1):57-64.

3. Burgess JO, Walker RS, Porche CJ, Rappold AJ. Light curing - an update. Compend Contin Educ Dent. 2002;23(10):889-92.

4. Caughman WF, Caughman GB, Shiflett RA, Rueggeberg FA, Schuster GS. Correlation of citotoxicity, filler loading and curing time of dental composites. Biomaterials 1991;12(8):737-40.

5. Dunn WJ, Bush AC. A comparison of polymerization by light emitting diode and halogen-based light-curing units. J Am Dent Assoc. 2002;133(3):335-41

6. Fano L, Ma WY, Marcoli PA, Pizzi S, Fano V. Polymerization of dental composite resins using plasma light. Biomaterials. 2002;23(4):1011-5.

7. Ferracane JL. Elution of leachable components from composites. J Oral Rehabil. 1994;21(4):441-52.

8. Ferracane JL, Mitchem JC, Condon JR, Todd R. Wear and marginal breakdown of composites with various degree of cure. J Dent Res. 1997;76(8):1508-16.

9. Guiraldo RD, Consani S, Lympius T, Schneider LFJ, Sinhoreti MAC, Correr-Sobrinho L. Influence of the light curing unit and thickness of residual dentin on generation of heat during composite photoactivation. J Oral Sci. 2008;50(2):137-42.

10. Hansen EK, Asmussen E. Reliability of three dental radiometers. Scand J Dent Res. 1993;101(2):115-9.

11. Harris JS, Jacobsen PH, O`Doherty DM. The effect of curing light intensity and test temperature on the dynamic mechanical properties o two polymer composites. J Oral Rehab. 1999;26(8):635-9.

12. Hofmann N, Hugo B, Schubert K, Klaiber B Comparison between a plasma arc light source and conventional halogen curing units regarding flexural strength, modulus, and hardness of photoactivated resin composites. Clin Oral Investig. 2000;4(3):140-7.

13. International Organization for Standardization (ISO). Norna ISO/TS: 10650: 1999 (E).

14. Masutani S, Setcos JC, Schinell RJ, Philips RW. Temperature rise during polymerization of visible light-activated composite resins Dent Mater. 1988;4(4):174-8.

15. McCabe JF. Cure performance of light-activated-composites by differential thermal analysis (DTA). Dent Mater. 1985;1(6):231-4.

16. Mills RW, Jandt KD, Ashworth SH. Dental composite depth of cure with halogen and blue light emitting diode technology. Br Dent J. 1999;186(8):388-91.
17. Munksgaard EC, Peutzfeldt A, Asmussen E. Elution o TEGDMA and BisGMA rom a resin and a resin composite cured with halogen or plasma light. Eur J Oral Sci. 2000;108(4):341-5.

18. Nomoto R, McCabe JF, Hirano S. Effect of aperture size on irradiance of LED curing units. Dent Mater. 2004;20(7):687-92.

19. Parr GR, Rueggeberg FA. Spectral analysis of commercial LED dental curing lights. J Dent Res. 2002;81(Spec Iss A) Abstract \#507:88

20. Peutzfeldt A, Sahafi A, Asmussen E. Characterization of resin composites polymerized with plasma arc curing units. Dent Mater 2000;16(5):330-6.

21. Price RBT, Felix CA and Andreou P. Knoop hardness of ten resin composites irradiated with high-power LED and quartz-tungsten-halogen lights. Biomaterials 2005;26(15):2631-41.

22. Rueggeberg FA. Contemporary issues in photocuring. Compend Contin Educ Dent. 1999;20(Suppl 25):S4-S15.

23. Ruyter IE, Øysæd H. Conversion in different depths of ultraviolet and visible light activated composite materials. Acta Odontol Scand. 1982;40(3):179-92.

24. Sahafi A, Peutzfeldt A, Asmussen E. Soft-start polymerization and marginal gap formation in vitro. Am J Dent. 2001;14(3):145-7.

25. Shortall AC, Harrington E, Wilson HJ. Light curing unit effectiveness assessed by dental radiometers. J Dent. 1995;23(4):227-32 .

26. Soh MS, Yap AU, Siow KS. Effectiveness of composite cure associated with different curing modes of LED lights. Oper Dent. 2003;28(4):371-7.

27. Uhl A, Mills RW, Jandt KD. Polymerization and light-induced heat of dental composites cured with LED and halogen technology. Biomaterials 2003;24(10):1809-20.

Recebido em 23/07/08 Aprovado em 18/09/08

Correspondência

Prof. Dr. Simonides Consan

Departamento de Odontologia Restauradora - Área Materiais Dentários Faculdade de Odontologia de Piracicaba Universidade Estadual de Campinas Av. Limeira, 901

CEP: 13414-903 - Piracicaba - SP - Brasil e-mail: consani@fop.unicamp.br 